\title{
Aktuelle Situation der homöopathischen Versorgung von Querschnittgelähmten in der Schweiz
}

\author{
Jürgen Pannek ${ }^{\mathrm{a}}$ Susanne Pannek-Rademacher ${ }^{\mathrm{b}}$ Martine Cachin-Jus ${ }^{\mathrm{c}}$ \\ a Neuro-Urologie, Schweizer Paraplegiker-Zentrum Nottwil, Nottwil, Schweiz; \\ ${ }^{b}$ Homöopathie Pannek, Basel, Schweiz; \\ 'SHI Praxis, Zug, Schweiz
}

Schlüsselwörter

Querschnittlähmung · Homöopathie · Medizinische Versorgung

\section{Zusammenfassung}

Hintergrund: Bis heute ist eine Querschnittlähmung (QSL) nicht kurativ behandelbar. Obwohl eine additive homöopathische Therapie der Sekundärfolgen einer QSL dokumentiert erfolgreich ist und eine hohe Nachfrage seitens der Betroffenen besteht, existiert in der Schweiz keine systematische homöopathische Versorgung für diese Patientengruppe. Mögliche Ursachen hierfür sind eine mangelnde Barrierefreiheit der Einrichtungen oder fehlende Ausbildung der Therapeuten. Methoden: Mittels eines standardisierten Fragebogens wurden alle in Fachverbänden registrierten homöopathischen Therapeuten in der Schweiz bezüglich Barrierefreiheit, Anzahl von Patienten mit QSL, medizinischer Versorgung und Fortbildungsbedarf befragt. Ergebnisse: 108 Fragebögen waren auswertbar. Obwohl 68 Therapeuten eine barrierefreie Praxis besassen, haben lediglich 31 Therapeuten im Jahr 2015 QSL-Patienten behandelt. In drei Einrichtungen wurden pro Jahr mehr als 5 Patienten mit QSL behandelt. 74 Personen (69\%) hatten einen Fortbildungsbedarf (65\% homöopathische Therapie, 31\% konventionelle Medizin). Offensichtlich wird lediglich ein geringer Teil der Personen mit QSL in der Schweiz homöopathisch betreut. Dabei konzentriert sich die Betreuung auf wenige Einrichtungen. Bauliche Hindernisse scheinen die Versorgung dieser Patientengruppe weniger einzuschränken als fehlende Fachkenntnisse. Aufgrund der komplexen Versorgung dieser Patientengruppe besteht ein erheblicher Fortbildungsbedarf. Schlussfolgerungen: Um die homöopathische Versorgung von Patienten mit QSL in der Schweiz zu optimieren, sind ein Netzwerk qualifizierter Homöopathen, Referenzzentren für komplexe Probleme, eine intensive Kooperation mit den konventionell medizinischen Therapeuten und eine gute Information der Betroffenen über die Grenzen und Möglichkeiten der Homöopathie vonnöten.

๑ 2017 S. Karger GmbH, Freiburg
Keywords

Spinal cord injury $\cdot$ Homeopathy $\cdot$ Health care

\section{Summary}

Current Situation of Homeopathic Care for Patients with Paraplegia in Switzerland

Background: Currently, there is no cure for spinal cord injury ( $\mathrm{SCl})$. Although adjunctive homeopathic treatment of the secondary complications of $\mathrm{SCl}$ has been proven to be effective and there is a high demand from the affected persons, no systematic homeopathic care for this patient group does exist in Switzerland. Possible reasons are either a limited accessibility of the treatment institutions or a lack of medical knowledge. Methods: With the help of the professional associations for homeopaths in Switzerland, all registered homeopaths were identified and supplied with a standardized questionnaire. The questions focused on accessibility, number of SCl patients treated per year, medical treatment, and the need of professional training. Results: 108 questionnaires were evaluated. Although 68 homeopaths provided unlimited wheelchair access to their institutions, only 31 therapists treated SCI patients in 2015. Three institutions treated more than $5 \mathrm{SCl}$ patients per year. 74 persons $(69 \%)$ requested further professional training $(65 \%$ homeopathy, $31 \%$ conventional medicine). Merely a small percentage of $\mathrm{SCl}$ patients in Switzerland receives homeopathic care. Homeopathic care is limited to a small number of institutions and seems to be limited rather by lack of medical training than by limited wheelchair access. Due to the complex medical care of these patients, a substantial demand for medical training exists. Conclusions: To optimize homeopathic care for $\mathrm{SCl}$ patients in Switzerland, a network of trained homeopaths, referral centers for complex problems, an intensive cooperation with conventional medical therapists, und an adequate information of the affected persons about the possibilities and limits of homeopathy are needed.

\section{KARGER}

(๑) 2017 S. Karger GmbH, Freiburg
Prof. Dr. med. Jürgen Pannek

Neuro-Urologie

Schweizer Paraplegiker-Zentrum

Guido A. Zäch Strasse 1, 6207 Nottwil, Schweiz

juergen.pannek@paraplegie.ch 


\section{Einleitung}

Trotz aller Fortschritte in der Grundlagenforschung ist eine Querschnittlähmung (QSL) bisher nicht kurativ behandelbar. Daher fokussiert die medizinische Versorgung der Betroffenen auf die Vermeidung von Sekundärkomplikationen [1]. In den letzten Dekaden konnte durch Verbesserungen der konventionellen Therapie die Lebenserwartung der Betroffenen deutlich verbessert werden; weiterhin wird die Lebensqualität jedoch häufig durch Sekundärkomplikationen eingeschränkt [2].

Die konventionelle medizinische Therapie dieser Komplikationen, vor allem Schmerzen, Spastik, Harnwegsinfekte sowie Blasen- und Darmfunktionsstörungen, ist nicht immer befriedigend [3]. Daher nimmt die Mehrzahl der Betroffenen komplementärmedizinische Behandlungen in Anspruch. Homöopathie und Akupunktur werden mit je 20\% aller Betroffenen am häufigsten angewandt [4] . Auch wenn die Zufriedenheit mit der Homöopathie bei den Patienten hoch ist, so wird die Mehrzahl der Betroffenen nicht homöopathisch versorgt. Während die konventionell medizinische Versorgung dieser Patienten in der Schweiz überwiegend in einem der vier Schweizer Querschnittzentren (Balgrist, Basel, Nottwil, Sion) erfolgt, existiert kein strukturiertes Angebot einer homöopathischen Versorgung für diese Patienten.

Die suboptimale Versorgung dieser Gruppe trotz dokumentiertem Interesse der Patienten hat viele mögliche Ursachen, wie z.B. eine fehlende Barrierefreiheit der Behandlungseinrichtungen oder fehlende medizinische Kenntnisse/Unsicherheit der Therapeuten bezüglich der QSL.

Daher sollte erfasst werden, wie viele Behandler in der Schweiz Personen mit QSL homöopathisch betreuen. Falls eine Praxis keine Querschnittgelähmten behandelt, sollen die Ursachen hierfür evaluiert und mögliche Probleme in der Behandlung dieser Patientengruppe evaluiert werden.

\section{Material und Methoden}

In Zusammenarbeit mit den entsprechenden Berufsverbänden (Schweizerischer Verein homöopathischer Ärztinnen und Ärzte, Homöopathieverband Schweiz) wurde allen in Berufsverbänden organisierten Homöopathen, sowohl ärztlich als auch nichtärztlich, ein standardisierter Fragebogen zugestellt. Dieser umfasste Fragen zur Barrierefreiheit der Einrichtung, ob und wie viele Patienten mit QSL im letzten Jahr behandelt worden sind, ob das für die QSL verantwortliche Trauma bei der Mittelfindung mit berücksichtigt wurde, welche Schwierigkeiten bei der Wahl des Mittels bestanden und ob ein Fortbildungsbedarf, homöopathisch und/oder konventionell medizinisch, bestand.

Nach 4 Wochen wurde die Anfrage bei den angeschriebenen Personen, welche nicht geantwortet hatten, wiederholt.

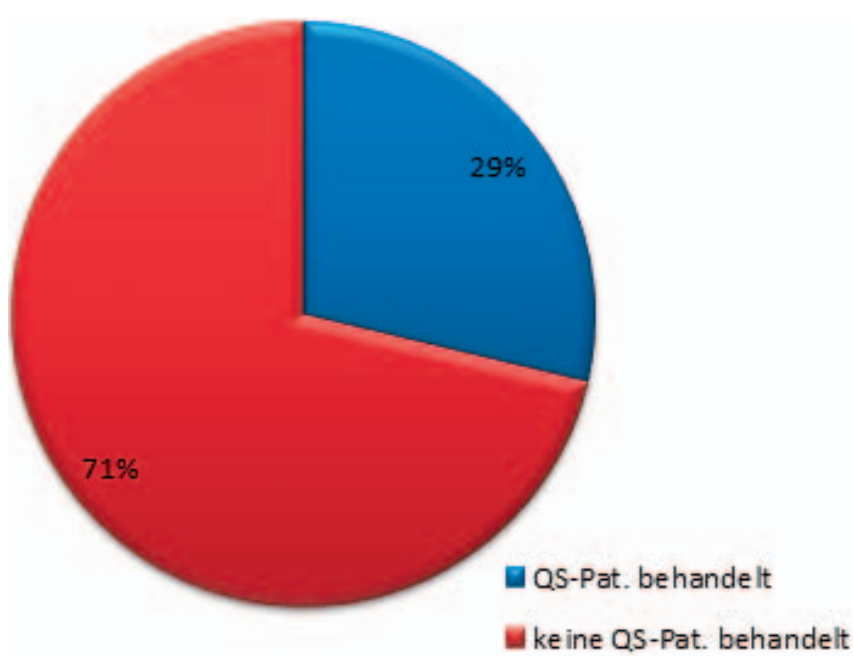

Abb. 1. Verteilung der homöopathischen Behandlung von Patienten mit QSL $(\mathrm{n}=108)$ in 2015 .

\section{Ergebnisse}

Insgesamt wurden anhand der Datenbanken der Berufs- und Standesorganisationen 300 ärztliche Homöopathen und 400 nichtärztliche Homöopathen angeschrieben. Davon haben 108 Therapeuten $(15,4 \%)$ geantwortet, deren Rückmeldungen die Basis der folgenden Auswertung bilden.

\section{Behandlung von Patienten mit Querschnittlähmung}

Von den 108 teilnehmenden Therapeuten gaben 68 an, eine barrierefreie Praxis zu besitzen.

31 Therapeuten (29\% der Teilnehmer, 45,6\% aller Teilnehmer mit barrierefreier Praxis) haben im Jahr 2015 QSL-Patienten behandelt; 77 Homöopathen haben keine QSL-Patienten behandelt (Abb. 1).

Von den 31 Institutionen/Praxen, die Patienten mit QSL behandelt haben, wurden in drei Einrichtungen mehr als 5 Patienten aus dieser Patientengruppe behandelt; in 22 Praxen wurde jeweils lediglich 1 Patient mit QSL betreut (Abb. 2).

\section{Therapie}

Wie bei der konventionell medizinischen Betreuung dieser Patientengruppe stellten Spastik, Schmerzen und Harnwegsinfekte die drei häufigsten Behandlungsindikationen dar (Abb. 3).

Bei der homöopathischen Fallaufnahme haben 88\% der Therapeuten Fragen nach dem Unfallhergang/schädigenden Ereignis, das zur QSL führte, gestellt, und bei $82 \%$ der Behandlungen wurden direkt Folgen des Unfalls/schädigenden Ereignisses behandelt. 


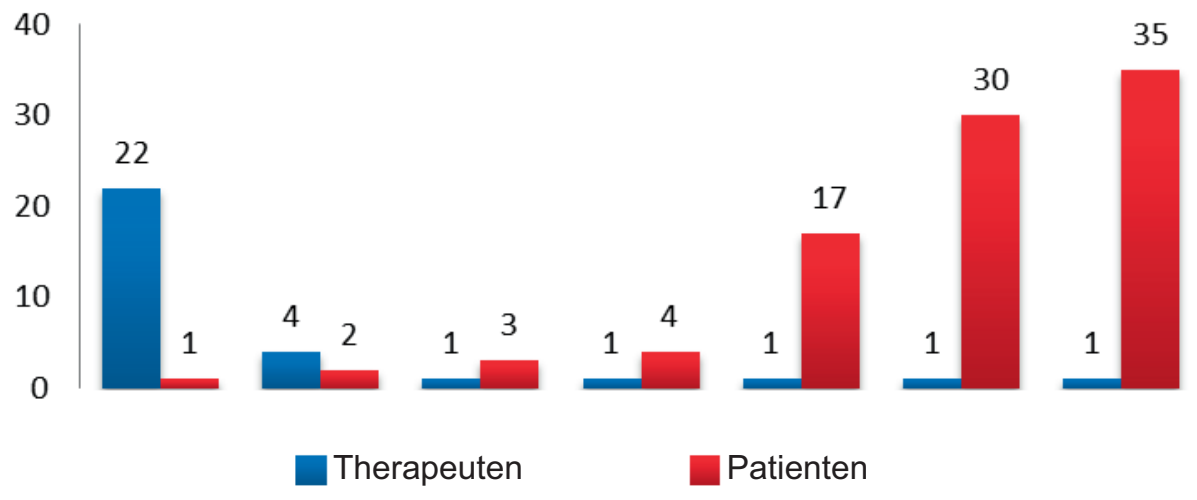

Abb. 2. Anzah
pro Therapeut.

Therapeuten

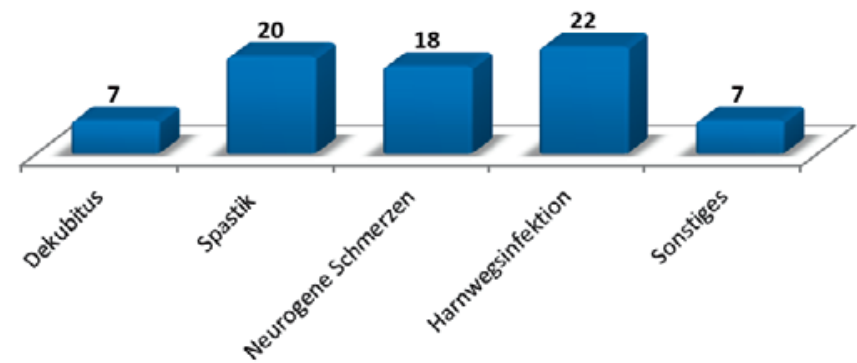

\section{Mittelwahl}

Fast die Hälfte der Therapeuten $(\mathrm{n}=16 ; 48 \%)$ gaben Probleme bei der Mittelfindung an, da aufgrund der sensomotorischen Ausfälle nach QSL Symptome oft fehlten oder nur spärlich vorhanden waren. Besonders die Prophylaxe und Therapie von Harnwegsinfekten stellten eine grosse Herausforderung dar.

Mehrere Therapeuten erwähnten, dass eine häufige Wiederholung desselben homöopathischen Mittels sowie die Verwendung von LM/Q-Potenzen eine bessere Wirkung bei diesen Patienten erzielten. Einmal wurde auch auf die bessere Wirkung von höheren Potenzen hingewiesen.

\section{Fortbildungsbedarf Therapeuten}

Von den befragten Therapeuten gaben 74 Personen (69\%) an, einen Fortbildungsbedarf zu haben. 65\% sahen einen Bedarf bei der homöopathischen Therapie von $\mathrm{Pa}$ tienten mit QSL; 31\% wünschten sich Informationen auf dem Gebiet der Rehabilitationsmedizin bzw. konventionellen Medizin.

\section{Diskussion}

Offensichtlich wird lediglich ein geringer Teil der ca. 8000 Personen mit QSL in der Schweiz homöopathisch betreut. Dabei konzentriert sich die Betreuung auf wenige Einrichtungen. Bauliche Hindernisse scheinen die Versorgung dieser Patientengruppe weniger einzuschränken als fehlende Fachkenntnisse.

Die medizinische Versorgung von Personen mit QSL ist komplex, interdisziplinär und interprofessionell [5]. Neben Ärzten für physikalische Medizin und Rehabilitation sind Intensivmediziner, Urologen, Orthopäden, Schmerzmediziner, plastische Chirurgen, Gastroenterologen sowie Ergo- und Physiotherapeuten regelmässig in die Versorgung von Personen mit QSL involviert - je nach Bedarf müssen weitere Berufsgruppen hinzugezogen werden. Da die Folgen der QSL zu neurogenen Funktionsstö-

Abb. 3. Behandlungsindikationen. (Sonstiges $=$ Depressionen, Folge von Schulterverletzungen, erektile Dysfunktion, Hautausschläge, Magen- und Darmbeschwerden, Prostatitis, Hämaturie und Kopfschmerzen.)

rungen führen, benötigen auch die Fachärzte der verschiedenen Disziplinen eine besondere Ausbildung innerhalb ihrer Fachrichtung. Um diesem Umstand Rechnung zu tragen, ist z.B. in der Urologie der Schwerpunkttitel Neuro-Urologie eingerichtet worden.

Daher benötigt auch ein Homöopath, der sich mit Personen mit QSL beschäftigt, besondere Kenntnisse, um z.B. bei der Mittelfindung entscheiden zu können, ob ein spezifisches Symptom pathognomonisch ist oder zur Mittelwahl herangezogen werden kann. Zudem können die Symptome lähmungsbedingt unspezifisch sein oder fehlen.

Die Ergebnisse unserer Studie reflektieren diese Situation. Weniger als die Hälfte aller Praxen, die rollstuhlgängig sind, behandelt Patienten mit QSL. Auch die hohe Nachfrage nach sowohl spezifisch homöopathischer als auch konventionell medizinischer Fortbildung unterstreicht die Notwendigkeit von Weiterbildung zu diesem Thema.

Andererseits wird die Zahl der heute in der Schweiz lebenden Personen mit QSL auf 8000-12 000 geschätzt. Diese Klientel stellt also nur eine relativ kleine Gruppe an Patienten dar, die jedoch einer komplexen Versorgung bedarf.

Somit erscheint eine generelle, umfassende Weiterbildung aller Homöopathen in der Schweiz nicht sinnvoll. Basierend auf den gewonnenen Erkenntnissen könnte eine Strategie zur Optimierung der homöopathischen Betreuung dieser Patientengruppe entsprechend der konventionell medizinischen Versorgung aufgebaut sein. In 
diesem Fall würde sich die Versorgung auf wenige, spezifisch ausgebildete Zentren beschränken. Diejenigen Zentren, die bereits die grösste Erfahrung im Umgang mit Personen mit QSL besitzen, können hierbei als Ausbildungszentren für andere Therapeuten dienen, bis eine flächendeckende Versorgung gewährleistet ist. Dabei wäre eine Option, eine enge Kooperation mit den entsprechenden Rehabilitationseinrichtungen zu etablieren. Diese Kooperation kann sowohl die medizinische Ausbildung der Therapeuten mit regelmässigen Weiterbildungsveranstaltungen beinhalten als auch eine Möglichkeit bieten, Betroffenen in einem grossen Ausmass systematisch und strukturiert Informationen über Homöopathie bei QSL zukommen zu lassen.

Eine Limitation der Studie ist der prozentual niedrige Rücklauf der Fragebögen, der sich auch durch weitere Nachfragen nicht erhöhen liess. Dennoch bildet unserer Meinung nach die Erhebung eine ausreichend solide Grundlage, welche die Ableitung einer Strategie ermöglicht. Vergleichende Erhebungen aus anderen Ländern haben wir nicht finden können.

Nicht evaluiert wurde bei dieser Studie die Bereitschaft von konventionell tätigen medizinischen Therapeuten, die Homöopathie mit in die Versorgung der Patienten einzubeziehen. Unsere Erfahrungen im Alltag zeigen nicht unerwartet ein weites Spektrum an Reaktionen von grossem Interesse bis zu strikter Ablehnung. Es ist kein Zufall, dass die erste homöopathische Sprechstunde für Querschnittgelähmte an einem Querschnittzentrum eingerichtet worden ist, das eine ganzheitliche Versorgung und eine Therapie nach einem ethno-bio-psychosozialen Modell (International Classification of Functioning, Disability and Health (ICF); basierend auf den Parametern Aktivität und Partizipation, Körperfunktionen und -strukturen, Umweltfaktoren, personenbezogene und Kontextfaktoren) etabliert hat [5]. Erste Erfahrungen mit dieser Kooperation zeigen ermutigende Resultate, sowohl bei urologischen Infektionen $[6,7]$ als auch bei Darmfunktionsstörungen [8], sowie eine hohe Akzeptanz und grosse Nachfrage bei den Betroffenen [4].

Aktuell existiert an einem der vier Rehabilitationszentren für Personen mit QSL in der Schweiz eine homöopathische Sprechstunde, während sich an einem zweiten eine solche im Aufbau befindet. Ziel ist es, an allen Zentren eine derartige Versorgung anzubieten.

In Analogie zur konventionell medizinischen Behandlung, bei der gravierende Probleme an den Zentren behandelt werden, die Patienten aber bei Bagatellbeschwerden vom Hausarzt versorgt werden, wäre auch eine Basisschulung für eine grosse Gruppe von Homöopathen sinnvoll, um eine heimatnahe Versorgung der Patienten gewährleisten zu können. Neben der Barrierefreiheit sind ein Netzwerk qualifizierter Homöopathen, Referenzzentren für komplexe Probleme, eine intensive Kooperation mit den konventionell medizinischen Therapeuten und eine gute Information der Betroffenen über die Grenzen und Möglichkeiten der Homöopathie unabdingbare Voraussetzungen für eine optimierte Versorgung. Als erste Schritte sind die Organisation von Fortbildungsveranstaltungen und der Aufbau von homöopathischen Konsiliardiensten an den entsprechenden Querschnittzentren geplant.

\section{Disclosure Statement}

Die Autoren bedanken sich bei der Homöopathie-Stiftung des Deutschen Zentralvereins homöopathischer Ärzte, welche diese Studie finanziell unterstützt hat.

\section{Literatur}

1 van den Berg ME, Castellote JM, de PedroCuesta J, Mahillo-Fernandez I: Survival after spinal cord injury: a systematic review. J Neurotrauma 2010;27:1517-1528.

2 Chiodo AE, Scelza WM, Kirshblum SC, Wuermser LA, Ho CH, Priebe MM: Spinal cord injury medicine. 5 . Long-term medical issues and health maintenance. Arch Phys Med Rehabil 2007;88(suppl 1):S76-83.

3 Pannek J: Treatment of urinary tract infection in persons with spinal cord injury: guidelines, evidence, and clinical practice. A questionnaire-based survey and review of the literature. J Spinal Cord Med 2011;34:11-15.
4 Pannek J, Pannek-Rademacher S, Wöllner J: Use of complementary and alternative medicine in persons with spinal cord injury in Switzerland: a survey study. Spinal Cord 2015;53:569-572.

5 Scheuringer M, Kirchberger I, Boldt C, EriksHoogland I, Rauch A, Velstra IM, Cieza A Identification of problems in individuals with spinal cord injury from the health professional perspective using the ICF: a worldwide expert survey. Spinal Cord 2010;48:529-536.
6 Pannek J, Pannek-Rademacher S, Jus MC, Jus MS: Usefulness of classical homoeopathy for the prevention of urinary tract infections in patients with neurogenic bladder dysfunction: a case series. Indian J Res Homoeopathy 2014;8:31-36.

7 Pannek J, Pannek-Rademacher S, Cachin-Jus $\mathrm{M}$ : Organ-preserving treatment of an epididymal abscess in a patient with spinal cord injury. Spinal Cord 2014;52(suppl 1):S7-8.

8 Pannek J, Pannek-Rademacher S, Jus MC, Jus MS: Paralytischer Ileus nach Ileum-Augmentation bei einem querschnittgelähmten Patienten - kann Homöopathie helfen? Urologe A $2014 ; 53: 1661-1663$. 